\title{
ccoative
commons
}

ISSN 2590-9770

The Art of Discrete and Applied Mathematics 2 (2019) \#P2.05

https://doi.org/10.26493/2590-9770.1305.f45

(Also available at http://adam-journal.eu)

\section{On the coset structure of distributive skew lattices}

\author{
João Pita Costa * \\ Jožef Stefan Institute, Ljubljana, Slovenia \\ Jonathan Leech \\ Westmont College, Santa Barbara, CA, USA
}

Received 14 July 2019, accepted 19 September 2019, published online 29 December 2019

\begin{abstract}
In the latest developments in the theory of skew lattices, the study of distributivity has been one of the main topics. The largest classes of examples of skew lattices thus far encountered are distributive. In this paper, we will discuss several aspects of distributivity in the absence of commutativity, and review recent related results in the context of the coset structure of skew lattices. We show that the coset perspective is essential to fully understand the nature of skew lattices and distributivity in particular. We will also discuss the combinatorial implications of these results and their impact in the study of skew lattices.
\end{abstract}

Keywords: Skew lattices, distributive structures, noncommutative structures, ordered structures, bands of semigroups.

Math. Subj. Class.: 06A75, 06B20, 06B75

\section{Introduction}

The study of skew lattices provides two perspectives that complement each other: in one perspective skew lattices are seen to be noncommutative variants of lattices; in the other they are viewed as double bands, two band structures occupying the same set, but are in some way dual to each other. Due to this, many of its basic concepts originate in either lattice theory or semigroup theory. Thus, e.g., skew lattices have a natural partial order similar to that occurring in lattice theory, but also the Green's equivalences that are fundamental in the study of bands. In recent developments in skew lattice theory, distributivity has been

\footnotetext{
* The author thanks the support of the Croatian Science Foundation's funding of the project EVOSOFT, with the reference UIP-2014-09-7945.

E-mail addresses: joao.pitacosta@ijs.si (João Pita Costa), leech@westmont.edu (Jonathan Leech)
}

()( This work is licensed under https://creativecommons.org/licenses/by/4.0/ 
one of the main topics [16]. When commutativity is no longer assumed, the concept of distributivity, inherited from lattice theory, has broken into various types of distributivity. Following Leech's early papers, and then in more recent publications, a group of mathematicians (K. Cvetko-Vah, M. Kinyon, J. Leech, M. Spinks and J. Pita Costa) have studied this topic. Much of this more recent study involves coset analysis which underlies the study of skew lattice architecture that was initiated by Leech in [15], and extended more recently by the researchers above, especially in [2, 6] and [10]. It is our aim to give a clear and concise overview of this work, while adding a few new results on the topic.

\section{Preliminaries}

A skew lattice is a set $S$ with binary operations $\wedge$ and $\vee$ that are both idempotent and associative, and satisfy the absorption laws $x \wedge(x \vee y)=x=(y \vee x) \wedge x$ and their $\vee-\wedge$ duals. Given that $\wedge$ and $\vee$ are idempotent and associative, these laws are equivalent to the absorption dualities: $x \wedge y=x$ iff $x \vee y=y$ and its $\vee-\wedge$ dual. A band is a semigroup consisting of just idempotents, and a semilattice is a commutative band. When $\mathbf{S}$ is a commutative semigroup, the set $E(S)$ of all idempotents in $\mathbf{S}$ is a semilattice under the semigroup multiplication. When $\mathbf{S}$ is not commutative, $E(S)$ need not be closed under multiplication [8]. Recall that a band is regular if it satisfies the identity $x y x z x=x y z x$. Skew lattices can be seen as double regular bands since both band reducts $(S, \wedge)$ and $(S, \vee)$ are regular. Green's relations are basic equivalence relations on semigroups, introduced in [7]. For bands they are defined by: $x \mathcal{D} y$ iff $x y x=x$ and $y x y=y ; x \mathcal{L} y$ iff $x y=x$ and $y x=y$; and $x \mathcal{R} y$ iff $x y=y$ and $y x=x$. Due to the absorption dualities, the Green's relations were defined for skew lattices in [12] by $\mathcal{R}=\mathcal{R}_{\wedge}=\mathcal{L}_{\vee}, \mathcal{L}=\mathcal{L}_{\wedge}=\mathcal{R}_{\vee}$ and $\mathcal{D}=\mathcal{D}_{\wedge}=\mathcal{D}_{\vee}$. In the literature, $\mathcal{D}$ is often called the natural equivalence. Right-handed skew lattices are the skew lattices for which $\mathcal{D}=\mathcal{R}$ while left-handed skew lattices satisfy $\mathcal{D}=\mathcal{L}$ [16]. Influenced by the natural quasiorders defined on bands [8], we define for skew lattices the following distinct concepts:

(i) the natural partial order defined by $x \geq y$ if $x \wedge y=y=y \wedge x$ or, dually, $x \vee y=$ $x=y \vee x$;

(ii) the natural preorder defined by $x \succeq y$ if $y \wedge x \wedge y=y$ or, dually, $x \vee y \vee x=x$.

Observe that $x \mathcal{D} y$ iff $x \succeq y$ and $y \succeq x$.

A band $\mathbf{S}$ is rectangular if $x y x=x$ holds. A skew lattice is rectangular if both band reducts $(S, \wedge)$ and $(S, \vee)$ are rectangular. This is equivalent to $x \wedge y=y \vee x$ holding. For every skew lattice $\mathbf{S}, \mathcal{D}$ is a congruence, $\mathbf{S} / \mathcal{D}$ is the maximal lattice image of $\mathbf{S}$ and all congruence classes of $\mathcal{D}$ are maximal rectangular skew lattices in $\mathbf{S}$. Recall that a chain (or totally ordered set) is an ordered set where every pair of elements are (order) related, and an antichain is an ordered set where no two elements are (order) related. We call $\mathbf{S}$ a skew chain whenever $S / \mathcal{D}$ is a chain. All $\mathcal{D}$-classes are antichains.

A primitive skew lattice is a skew lattice $\mathbf{S}$ composed of two comparable $\mathcal{D}$-classes $A$ and $B$ that are comparable in that $A \geq B$ in $S / \mathcal{D}, a \succeq b$ for all $a \in A$ and $b \in B$. A skew diamond is a skew lattice composed by two incomparable $\mathcal{D}$-classes, $A$ and $B$, a join $\mathcal{D}$-class $J=A \vee B$ and a meet $\mathcal{D}$-class $M=A \wedge B$. In particular, we express a primitive skew lattice as $\{A>B\}$ and the skew diamond $\mathbf{X}$ as $\{J>A, B>M\}$. Note that if $A$ and $B$ are incomparable $\mathcal{D}$-classes in a skew lattice $\mathbf{S}$, with $A \wedge B=M$ and $A \vee B=J$, then the $\mathcal{D}$-relation on the skew diamond $\mathbf{X}=\{J>A, B>M\}$ is the restriction of the 
$\mathcal{D}$-relation on $\mathbf{S}$, and the $\mathcal{D}$-classes in the sub skew lattice $\mathbf{X}$ are exactly $A, B, M$ and $J$. In this case $\{J>A\},\{J>B\},\{A>M\}$ and $\{B>M\}$ are primitive skew lattices.

Proposition 2.1 ([12]). Let $A$ and $B$ be comparable $\mathcal{D}$-classes in a skew lattice $\mathbf{S}$ such that $A \geq B$. Then, for each $a \in A$, there exists $b \in B$ such that $a \geq b$, and dually, for each $b \in B$, there exists $a \in A$ such that $a \geq b$.

Proposition 2.2 ([12]). Let $\{J>A, B>M\}$ be a skew diamond. Then, for every $a \in A$ there exists $b \in B$ such that $a \vee b=b \vee a$ in $J$ and $a \wedge b=b \wedge$ in M. Moreover,

$$
\begin{aligned}
J & =\{a \vee b \mid a \in A, b \in B \text { and } a \vee b=b \vee a\} \quad \text { and } \\
M & =\{a \wedge b \mid a \in A, b \in B \text { and } a \wedge b=b \wedge a\} .
\end{aligned}
$$

The variety of distributive skew lattices was first introduced in [12] by the defining identities:

(d1) $x \wedge(y \vee z) \wedge x=(x \wedge y \wedge x) \vee(x \wedge z \wedge x)$,

(d2) $x \vee(y \wedge z) \vee x=(x \vee y \vee x) \wedge(x \vee z \vee x)$

Skew lattices satisfying $(\mathrm{d} 1)$ are called $\wedge$-distributive while skew lattices satisfying $(\mathrm{d} 2)$ are called $\vee$-distributive. Clearly, the lattice $\mathbf{S} / \mathcal{D}$ is distributive in either case, because the distributivity of $\wedge$ will imply the distributivity of $\vee$ in the presence of commutativity. Since $x \vee y=y \wedge x$ in any $\mathcal{D}$-class, it is easily seen that in any skew lattice both (d1) and (d2) must hold when $y$ and $z$ are $\mathcal{D}$-related. We thus also have the following pair of "balanced" identities that are equivalent to (d1) and (d2) respectively.

Proposition 2.3 ([19]). Let $\mathbf{S}$ be a skew lattice. Then $\mathbf{S}$ is distributive if and only if for all $a, b, c \in S$,

(i) $a \wedge(c \vee b \vee c) \wedge a=(a \wedge c \wedge a) \vee(a \wedge b \wedge a) \vee(a \wedge c \wedge a)$ and, dually,

(ii) $a \vee(c \wedge b \wedge c) \vee a=(a \vee c \vee a) \wedge(a \vee b \vee a) \wedge(a \vee c \vee a)$

In order to explore further the different concepts of distributivity occurring in the literature, consider the following axioms:

(d3) $x \wedge(y \vee z)=(x \wedge y) \vee(x \wedge z)$,

(d4) $(x \vee y) \wedge z=(x \wedge z) \vee(y \wedge z)$,

(d5) $x \vee(y \wedge z)=(x \vee y) \wedge(x \vee z)$,

(d6) $(x \wedge y) \vee z=(x \vee z) \wedge(y \vee z)$.

Skew lattices satisfying (d3) and (d4) are called strongly distributive while skew lattices satisfying (d5) and (d6) are called co-strongly distributive. Examples exist of skew lattices satisfying any combination of these four distributive identities (cf. [11]). A skew lattice satisfies all four distributive laws (d3) to (d6) if and only if it is the direct product of a rectangular skew lattice with a distributive lattice (cf. [11]). This is also equivalent to a skew lattice satisfying the following pair of identities:

(d7) $x \wedge(y \vee z) \wedge w=(x \wedge y \wedge w) \vee(x \wedge z \wedge w)$, 
(d8) $x \vee(y \wedge z) \vee w=(x \vee y \vee w) \wedge(x \vee z \vee w)$

Skew lattices satisfying (d7) are called $\wedge$-bidistributive skew lattices while skew lattices satisfying (d8) are called $\vee$-bidistributive skew lattices. Clearly a number of implications are immediate:

$$
((\mathrm{d} 3) \text { and }(\mathrm{d} 4)) \Rightarrow(\mathrm{d} 7) \Rightarrow(\mathrm{d} 1) \text {, while }((\mathrm{d} 5) \text { and }(\mathrm{d} 6)) \Rightarrow(\mathrm{d} 8) \Rightarrow(\mathrm{d} 2) \text {. }
$$

It turns out that (d7) implies both (d1) and (d2) as does (d8), and thus also (d3) and (d4) together, as well as (d5) and (d6) together [14]. For lattices, all of these identities reduce to either (d3) or (d5), which in turn are equivalent. A skew lattice $\mathbf{S}$ is quasi-distributive if its maximal lattice image $\mathbf{S} / \mathcal{D}$ is distributive. Clearly this is the case for the types of distributive skew lattices above. In general, a skew lattice is quasi-distributive precisely when no copy of either 5-element non-distributive lattice $\mathbf{M}_{5}$ or $\mathbf{N}_{5}$ is a subalgebra [4]. Finally, a skew lattice $\mathbf{S}$ is cancellative if for all $x, y, z \in S$,

$$
\begin{aligned}
& z \vee x=z \vee y \text { and } z \wedge x=z \wedge z \text { imply } x=y, \text { and } \\
& x \vee z=y \vee z \text { and } x \wedge z=y \wedge z \text { imply } x=y
\end{aligned}
$$

For lattices, being cancellative is equivalent to being distributive. In general, cancellative skew lattices are quasi-distributive, but need not be distributive. Skew lattices of idempotents in rings are always cancellative.

To understand further the connections between these variations on a distributive theme, we need several further concepts. A skew lattice is symmetric if commutativity is unambiguous in that for all $x, y \in S$,

$$
x \wedge y=y \wedge x \text { iff } x \vee y=y \vee x .
$$

Our interest in symmetric skew lattices is due in part to the fact that skew lattices of idempotents in rings are always distributive and symmetric.

A skew lattice $\mathbf{S}$ is normal if it satisfies

$$
x \wedge y \wedge z \wedge w=x \wedge z \wedge y \wedge w
$$

dually, if it satisfies

$$
x \vee y \vee z \vee w=x \vee z \vee y \vee w
$$

it is conormal. While (d1) and (d2) are not equivalent for skew lattices in general, for symmetric skew lattices we have:

Theorem 2.4 ([20, 3]). Given a symmetric skew lattice, (d1) holds if and only if (d2) holds.

Theorem 2.5 ([16]). A skew lattice is strongly distributive in that it satisfies (d3) and (d4) if and only if it is distributive, symmetric and normal. It is co-strongly distributive in that it satisfies (d5) and (d6) if and only if it is distributive, symmetric and conormal. It is $\wedge$-distributive in that it satisfies (d7) if and only if it is distributive and normal. It is $\vee$ distributive in that it satisfies (d8) if and only if it is distributive and conormal. Finally, it is both $\wedge$-distributive and $\vee$-distributive if and only if it is the product of a distributive lattice and a rectangular skew lattice. 
A skew Boolean algebra is an algebra $\mathbf{S}=(S ; \vee, \wedge, \backslash, 0)$ such that $(S ; \vee, \wedge, 0)$ is a distributive, normal and symmetric skew lattice with a constant 0 , called zero, satisfying $x \wedge 0=0=0 \wedge x$, and a binary operation $\backslash$ on $\mathbf{S}$, called relative complement, satisfying $(x \wedge y \wedge x) \vee(x \backslash y)=x$ and $(x \wedge y \wedge x) \wedge(x \backslash y)=0$. Skew lattice reducts of skew Boolean algebras are strongly distributive. In general, a skew lattice can be embedded in the skew lattice reduct of a skew Boolean algebra if and only if it is strongly distributive [13]. Given a ring $R$ whose set $E(R)$ of idempotents are closed under multiplication, then $E(R)$ forms a skew Boolean algebra under the operations:

$$
\begin{aligned}
& e \wedge f=e f \\
& e \vee f=e+f+f e-e f e-f e f, \\
& e \backslash f=e-e f e .
\end{aligned}
$$

It should be mentioned that strongly distributive skew lattices, and in particular skew Boolean algebras, are always cancellative.

Consider a skew lattice $\mathbf{S}$ consisting of exactly two $\mathcal{D}$-classes $A>B$. Given $b \in B$, the subset $A \wedge b \wedge A=\{a \wedge b \wedge a \mid a \in A\}$ of $B$ is said to be a coset of $A$ in $B$ (or an $A$-coset in $B$ ). Similarly, a coset of $B$ in $A$ (or a $B$-coset in $A$ ) is any subset $B \vee a \vee B=\{b \vee a \vee b \mid b \in B\}$ of $A$, for a fixed $a \in A$. On the other hand, given $a \in A$, the image set of $a$ in $B$ is the set $a \wedge B \wedge a=\{a \wedge b \wedge a \mid b \in B\}=\{b \in B \mid b<a\}$. Dually, given $b \in B$ the set $b \vee A \vee b=\{a \in A \mid b<a\}$ is the image set of $b$ in $A$ (cf. [15, 17, 19]).

Theorem 2.6 ([15]). Let $\mathbf{S}$ be a skew lattice with comparable $\mathcal{D}$-classes $A>B$. Then, $B$ is partitioned by the cosets of $A$ in $B$ and the image set in $B$ of any element $a \in A$ in $B$ is a transversal of the cosets of $A$ in $B$; dual remarks hold for any $b \in B$ and the cosets of $B$ in $A$ that determine a partition of $A$. Moreover, any coset $B \vee a \vee B$ of $B$ in $A$ is isomorphic to any coset $A \wedge b \wedge A$ of $A$ in $B$ under a natural bijection $\varphi$ defined implicitly for any $a \in A$ and $b \in B$ by: $x \in B \vee a \vee B$ corresponds to $y \in A \wedge b \wedge A$ if and only if $x \geq y$. Thus, all cosets in $A$ or $B$ are mutually isomorphic.

Furthermore, the operations $\wedge$ and $\vee$ on $A \cup B$ are determined jointly by the coset bijections and the rectangular structure of each $\mathcal{D}$-class. Even if $A$ and $B$ are unrelated so that one has a proper skew diamond, $\{A \vee B>A, B>A \wedge B\}$, coset bijections play an important role in calculating both $a \vee b$ in $A \vee B$ and $a \wedge b$ in $A \wedge B$. (See [15, Lemma 1.3 and Theorem 3.3].) Thus, even if the natural partial order does not completely determine the operations $\wedge$ and $\vee$ as it does for lattices, it still has a very significant role.

All cosets and all image sets are rectangular sub-skew lattices (cf. [17]). E.g., $(A \wedge$ $b \wedge A) \wedge\left(A \wedge b^{\prime} \wedge A\right)$ quickly reduces to $A \wedge b \wedge b^{\prime} \wedge A$ which in turn must also be $\left(A \wedge b^{\prime} \wedge A\right) \vee(A \wedge b \wedge A)$. Moreover, coset equality is given by the following result:

Proposition 2.7 ([6]). Let $\mathbf{S}$ be a skew lattice with comparable $\mathcal{D}$-classes $A>B$ and let $y, y^{\prime} \in B$. Then the following are equivalent:

(i) $A \wedge y \wedge A=A \wedge y^{\prime} \wedge A$;

(ii) for all $x \in A, x \wedge y \wedge x=x \wedge y^{\prime} \wedge x$;

(iii) there exists $x \in A$ such that $x \wedge y \wedge x=x \wedge y^{\prime} \wedge x$. 
Dual results hold for $B$-cosets in A, having similar statements.

The following propositions characterize important properties of skew lattices through coset structure flavored aspects of these algebras. They motivate the results in the context of coset laws characterization discussed later in this paper.

Proposition 2.8 ([15]). A skew lattice $\mathbf{S}$ is symmetric if and only if given any skew diamond $\{J>A, B>M\}$ in $\mathbf{S}$ and any $m, m^{\prime} \in M, j, j^{\prime} \in J$ the following equivalences hold:

(a) $J \wedge m \wedge J=J \wedge m^{\prime} \wedge J$ iff $A \wedge m \wedge A=A \wedge m^{\prime} \wedge A$ and $B \wedge m \wedge B=B \wedge m^{\prime} \wedge B$;

(b) $M \vee j \vee M=M \vee j^{\prime} \vee M$ iff $A \vee j \vee A=A \vee j^{\prime} \vee A$ and $B \vee j \vee B=B \vee j^{\prime} \vee B$.

Proposition 2.9 ([18]). A skew lattice $\mathbf{S}$ is normal iff for each comparable pair of $\mathcal{D}$ classes $A>B$ in $\mathbf{S}$ and all $x, x^{\prime} \in B, A \wedge x \wedge A=A \wedge x^{\prime} \wedge A$. Dually, $\mathbf{S}$ is conormal iff for all comparable pairs of D-classes $A>B$ in $\mathbf{S}$ and all $x, x^{\prime} \in A, B \vee x \vee B=B \vee x^{\prime} \vee B$.

Proposition 2.9 above essentially states that $\mathbf{S}$ is a normal skew lattice if and only if, for each comparable pair of $\mathcal{D}$-classes $A>B$ in $\mathbf{S}$ and for each $x \in A$, a unique $y \in B$ exists such that $y \leq x$. A dual result holds for conormal skew lattices.

\section{Coset bijections and categorical skew lattices}

A skew lattice is categorical if nonempty composites of coset bijections are coset bijections. That is, given $\mathcal{D}$-classes $A>B>C$ and coset bijections $\phi: B \vee a \vee B \rightarrow A \wedge b \wedge A$ and $\psi: C \vee b^{\prime} \vee S \rightarrow B \wedge c \wedge B$ with

$$
(A \wedge b \wedge A) \cap\left(C \vee b^{\prime} \vee S\right) \neq \emptyset
$$

then the resulting nonempty composite $\psi \phi$ is a bijection between cosets in $A$ and $C$. Rectangular and primitive skew lattices are trivially categorical. Skew lattices in rings and skew Boolean algebras provide nontrivial examples (cf. [15] and [1]). Clearly a skew lattice is categorical iff every skew chain of $\mathcal{D}$-classes $A>B>C$ in $\mathbf{S}$ is categorical. Indeed, this property is primarily about the skew chains of $\mathcal{D}$-classes in a skew lattice and about their coset bijections being well-behaved under composition. Upon adding empty partial bijections one indeed obtains a category of partial bijections whose objects are $\mathcal{D}$-classes and whose morphisms are given by coset bijections and the empty partial bijections.

Example 3.1. A minimal example of a non-categorical skew lattice is given by the 8element left-handed skew chain given in Figure 1. In this example, considering the skew chain $\{0,4\}>\{3,6,1,7\}>\{2,5\}$, the coset bijections are the following:

$$
\begin{array}{rlrl}
\varphi_{1}:\{0,4\} \rightarrow\{3,1\}, & \varphi_{2}:\{0,4\} \rightarrow\{6,7\} \\
\psi_{1}:\{3,7\} \rightarrow\{2,5\}, & \psi_{2}:\{6,1\} \rightarrow\{2,5\} \\
\chi:\{0,4\} \rightarrow\{2,5\} & &
\end{array}
$$

Observe that 0 has no image under $\psi_{2} \circ \varphi_{1}$ and that $\chi(0) \in\{2,5\}$ so that $\psi_{2} \circ \varphi_{1} \neq \chi$. The reader can find a detailed study of such examples in [9] where this skew lattice is called $\mathbf{X}_{2}$ and its right-handed version is called $\mathbf{Y}_{2}$. 


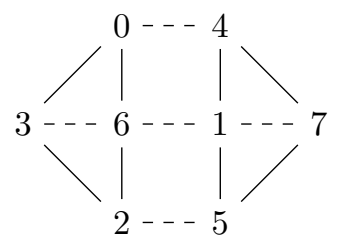

\begin{tabular}{|c|c|c|c|c|c|c|c|c|c|c|c|c|c|c|c|c|c|}
\hline$\wedge$ & 0 & 1 & 2 & 3 & 4 & 5 & 6 & 7 & V & 0 & 1 & 2 & 3 & 4 & 5 & 6 & 7 \\
\hline 0 & 0 & 3 & 2 & 3 & 0 & 2 & 6 & 6 & 0 & 0 & 4 & 0 & 0 & 4 & 4 & $\overline{0}$ & 4 \\
\hline 1 & 1 & 1 & 5 & 1 & 1 & 5 & 1 & 1 & 1 & 0 & 1 & 6 & 3 & 4 & 1 & 6 & 7 \\
\hline 2 & 2 & 2 & 2 & 2 & 2 & 2 & 2 & 2 & 2 & 0 & 1 & 2 & 3 & 4 & 5 & 6 & 7 \\
\hline 3 & 3 & 3 & 2 & 3 & 3 & 2 & 3 & 3 & 3 & 0 & 1 & 3 & 3 & 4 & 7 & 6 & 1 \\
\hline 4 & 4 & 1 & 5 & 1 & 4 & 5 & 7 & 7 & 4 & 0 & 4 & 0 & 0 & 4 & 4 & 0 & $I$ \\
\hline 5 & 5 & 5 & 5 & 5 & 5 & 5 & 5 & 5 & 5 & 0 & 1 & 2 & 3 & 4 & 5 & 6 & 7 \\
\hline 6 & 6 & 6 & 2 & 6 & 6 & 2 & 6 & 6 & 6 & 0 & 1 & 6 & 3 & 4 & 1 & 6 & 7 \\
\hline 7 & 7 & 7 & 5 & 7 & 7 & 5 & 7 & 7 & 7 & 0 & 1 & 3 & 3 & 4 & 7 & 6 & \\
\hline
\end{tabular}

Figure 1: The admissible Hasse diagram of a left-handed non-categorical skew lattice.

A categorical skew lattice is strictly categorical if the compositions of coset bijections between comparable $\mathcal{D}$-classes $A>B>C$ are never empty. Rectangular and primitive skew lattices, as well as skew Boolean algebras are strictly categorical skew lattices (cf. [1]). Normal and conormal skew lattices are strictly categorical (cf. [9]). As strictly categorical skew lattices form a variety [9], sub skew lattices of strictly categorical skew lattices are also strictly categorical. Clearly a skew lattice is strictly categorical if and only if every skew chain of $\mathcal{D}$-classes $A>B>C$ in $\mathbf{S}$ is strictly categorical. In the strictly categorical case, the above category of coset bijections can be defined without the need of empty partial bijections.

Example 3.2. A minimal example of a categorical skew lattice that is not strictly categorical is given by the right-handed manifestation of the skew chain with three $\mathcal{D}$-classes in Figure 2. In fact, the composition of the coset bijections $\psi:\{1\} \rightarrow\{a\}$ and $\varphi^{\prime}:\{b\} \rightarrow\{0\}$ is empty. Observe that $\chi:\{1\} \rightarrow\{0\}$ can be decomposed either by the composition of $\psi$ and $\varphi:\{a\} \rightarrow\{0\}$, or by the composition of $\psi^{\prime}:\{1\} \rightarrow\{b\}$ and $\varphi^{\prime}$ (cf. [9]).

Categorical skew lattices and strictly categorical skew lattices are subvarieties of the variety of skew lattices (cf. [16]) as are the many classes of skew lattices considered in the previous section. What follows is a practical characterization of strictly categorical skew chains, followed by an immediate application.

Proposition 3.3 ([9]). A skew chain $A>B>C$ is strictly categorical if and only if given $a \in A, b, b^{\prime} \in B$ and $c \in C$ such that $a>b>c$ and $a>b^{\prime}>c$, it follows that $b=b^{\prime}$.

Theorem 3.4. A strictly categorical skew lattice $\mathbf{S}$ is distributive if and only if it is quasidistributive, i.e., $\mathbf{S} / \mathcal{D}$ is distributive. In particular, a (co-)normal skew lattice is distributive if and only if it is quasi-distributive.

Proof. We need only verify $\Leftarrow$. Suppose $\mathbf{S}$ is quasi-distributive. Then for all $x, y, z \in S$,

$$
x \wedge(y \wedge z) \wedge x \mathcal{D}(x \wedge y \wedge x) \vee(x \wedge z \wedge x) .
$$




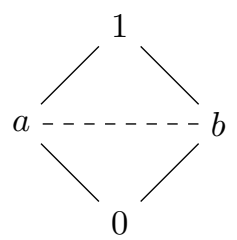

\begin{tabular}{|c|c|c|c|c|c|c|c|c|c|}
\hline$\wedge$ & 0 & $a$ & $b$ & 1 & V & 0 & $a$ & $b$ & 1 \\
\hline 0 & 0 & 0 & 0 & 0 & 0 & 0 & $a$ & $b$ & \\
\hline$a$ & 0 & $a$ & $b$ & $a$ & $a$ & $a$ & $a$ & $a$ & 1 \\
\hline$b$ & 0 & $a$ & $b$ & $b$ & $b$ & $b$ & $b$ & $b$ & 1 \\
\hline 1 & 0 & $a$ & $b$ & 1 & 1 & 1 & 1 & 1 & 1 \\
\hline
\end{tabular}

Figure 2: The Cayley tables and the admissible Hasse diagram of a non-strictly categorical right-handed skew lattice.

Clearly $x>x \wedge(y \vee z) \wedge x$ and $x>(x \wedge y \wedge x) \vee(x \wedge z \wedge x)$. On the other hand both of these polynomial terms are easily seen to be $>x \wedge z \wedge y \wedge x$. Thus by the above midpoint criterion, $x \wedge(y \vee z) \wedge x=(x \wedge y \wedge x) \vee(x \wedge z \wedge x)$. We illustrate the details for showing both terms being bigger than $x \wedge z \wedge y \wedge x$ by the following calculations:

$$
\begin{aligned}
x \wedge(y \vee z) \wedge x \wedge x \wedge z \wedge y \wedge x & =x \wedge(y \vee z) \wedge x \wedge z \wedge y \wedge x \\
& =x \wedge(y \vee z) \wedge z \wedge y \wedge x=x \wedge z \wedge y \wedge x \\
{[(x \wedge y \wedge x) \vee(x \wedge z \wedge x)] \wedge x } & \wedge z \wedge y \wedge x \\
& =[(x \wedge y \wedge x) \vee(x \wedge z \wedge x)] \wedge x \wedge z \wedge x \wedge y \wedge x \\
& =x \wedge z \wedge x \wedge y \wedge x=x \wedge z \wedge y \wedge x
\end{aligned}
$$

The dual identities are similarly verified.

Theorem 3.5 ([2]). A skew chain $\mathbf{S}$ consisting of $\mathcal{D}$-classes $A>B>C$ is categorical if and only if for all elements $a \in A, b \in B$ and $c \in C$ satisfying $a>b>c$, one (and hence both) of the following equivalent statements hold:

(i) $(A \wedge b \wedge A) \cap(C \vee b \vee C)=(C \vee a \vee C) \wedge b \wedge(C \vee a \vee C)$;

(ii) $(A \wedge b \wedge A) \cap(C \vee b \vee C)=(A \wedge c \wedge A) \vee b \vee(A \wedge c \wedge A)$.

Moreover, $\mathbf{S}$ is strictly categorical iff in addition to (i) and (ii),

$$
(A \wedge b \wedge A) \cap\left(C \vee b^{\prime} \vee C\right) \neq \emptyset
$$

for all $b, b^{\prime} \in B$.

In conformity with [10], a nonempty intersection $(A \wedge b \wedge A) \cap\left(C \vee b^{\prime} \vee C\right)$ of $A$-cosets and $C$-cosets in middle class $B$ is called an $A-C$ coset in $B$. Any such intersection equals $(A \wedge y \wedge A) \cap(C \vee y \vee C)$ for all $y$ in this intersection.

Let $A \geq B$ be comparable $\mathcal{D}$ classes in a normal skew lattice $\mathbf{S}$ and let $a \in A$. Due to the normality of $\mathbf{S}$, Proposition 2.9 implies that, for all $a \in A$ there exists a unique $b \in B$ 
such that $a \geq b$. Since $a \wedge b \wedge a \leq a$ for all $b \in B, b \leq a$ in $B$ if and only if $b=a \wedge b \wedge a$. Hence, given $b, b^{\prime} \in B$ such that $b, b^{\prime} \leq a$ in $B$, we have

$$
\begin{aligned}
b & =a \wedge b \wedge a=a \wedge b \wedge b^{\prime} \wedge b \wedge a=a \wedge b \wedge b^{\prime} \wedge b^{\prime} \wedge b \wedge a \\
& =a \wedge b^{\prime} \wedge b \wedge b \wedge b^{\prime} \wedge a=a \wedge b^{\prime} \wedge a=b^{\prime} .
\end{aligned}
$$

Thus, normal skew lattices are strictly categorical. Indeed, the lower $\mathcal{D}$-class in any maximal primitive sub skew lattice of a normal skew lattice has exactly one coset. Thus, adjacent coset bijections are closed under composition and in particular have nonempty compositions. Dual remarks hold for conormal skew lattices. Strictly categorical skew lattices, however, need not be either normal or conormal. What follows is an example of a

\begin{tabular}{|c|c|c|c|c|c|c|c|}
\hline$\wedge$ & $a$ & $b$ & 1 & $V$ & $a$ & $b$ & 1 \\
\hline$a$ & $a$ & $b$ & $a$ & $a$ & $a$ & $a$ & 1 \\
\hline$b$ & $a$ & $b$ & $b$ & $b$ & $b$ & $b$ & 1 \\
\hline 1 & $a$ & $b$ & 1 & 1 & 1 & 1 & 1 \\
\hline
\end{tabular}
conormal, but not normal, skew lattice.

Figure 3: The Cayley tables and the admissible Hasse diagram of a non-normal but strictly categorical right-handed skew lattice.

Example 3.6. Strictly categorical skew lattices need not be normal: the admissible Hasse diagram in Figure 3 represents a right-handed skew chain defined by the respective Cayley tables. It is strictly categorical but normality fails as the upper $\mathcal{D}$-class determines more then one coset in the lower $\mathcal{D}$-class.

Proposition 3.7 ([18]). Let $\mathbf{S}$ be a skew lattice. The following statements are equivalent:

(i) $\mathbf{S}$ is categorical;

(ii) for all distinct $\mathcal{D}$-classes $A>B>C$ with elements $b \in B$ and $c \in C$ that satisfy $b>c$, the coset bijection $\phi_{b, c}: C \vee b \vee C \rightarrow B \wedge c \wedge B$ restricted to the corresponding $A C$-coset $(A \wedge b \wedge A) \cap(C \vee b \vee C)$ in $B$ is a bijection of the later onto the $A$-coset $A \wedge c \wedge A$ in $C$;

(ii') for all distinct $\mathcal{D}$-classes $A>B>C$ with elements $a \in A$ and $b \in B$ that satisfy $a>b$, the coset bijection $\phi_{b, a}: A \wedge b \wedge A \rightarrow B \vee a \vee B$ restricted to the corresponding $A C$-coset $(A \wedge b \wedge A) \cap(C \vee b \vee C)$ in $B$ is a bijection of the latter onto the $C$-coset $C \vee a \vee C$ in $A$.

The following results, based on research of Kinyon and Leech on distributive skew lattices (cf. [9] and [10]), reveal the relationship between distributivity and (strict) categoricity, and allow us to extend the ideas in Proposition 3.7 to coset laws in the categorical case and in particular in the distributive case. 
Given a skew chain $A>B>C$, two elements $b, b^{\prime} \in B$ are $A C$-connected if a finite sequence $b=b_{0}, b_{1}, \ldots, b_{n}=b^{\prime}$ exists in $B$ such that $A \wedge b_{i} \wedge A=A \wedge b_{i+1} \wedge A$ or $C \vee b_{i} \vee C=C \vee b_{i+1} \vee C$ for all $0 \leq i \leq n-1$. Clearly all elements in an $A$-coset [C-coset] in $B$ are $A C$-connected.

Corollary 3.8. Given a categorical skew chain $A>B>C$, all $A C$-cosets in $B$ have the same size as all $A$-cosets in $C$ and all $C$-cosets in $A$. Conversely, given a skew chain $A>B>C$ having this property, if the common cardinal size is also finite, then the skew chain is categorical.

Given a skew chain $A>B>C$, an $A C$-component in $B$ is a maximal $A C$-connected subset of $B . B$ is $A C$-connected if all $b, b^{\prime}$ in it are $A C$-connected.

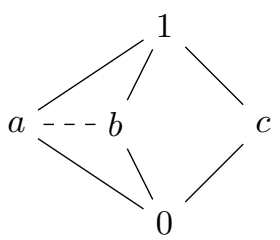

\begin{tabular}{c||ccccc}
$\wedge$ & 0 & $a$ & $b$ & $c$ & 1 \\
\hline \hline 0 & 0 & 0 & 0 & 0 & 0 \\
$a$ & 0 & $a$ & $b$ & 0 & $a$ \\
$b$ & 0 & $a$ & $b$ & 0 & $b$ \\
$c$ & 0 & 0 & 0 & $c$ & $c$ \\
1 & 0 & $a$ & $b$ & $c$ & 1
\end{tabular}

\begin{tabular}{c||ccccc}
$\vee$ & 0 & $a$ & $b$ & $c$ & 1 \\
\hline \hline 0 & 0 & $a$ & $b$ & $c$ & 1 \\
$a$ & $a$ & $a$ & $a$ & 1 & 1 \\
$b$ & $b$ & $b$ & $b$ & 1 & 1 \\
$c$ & $c$ & 1 & 1 & $c$ & 1 \\
1 & 1 & 1 & 1 & 1 & 1
\end{tabular}

Figure 4: The Cayley tables and admissible Hasse diagram of the right-handed skew lattice $\mathrm{NC}_{5}^{R}$.

Example 3.9. For instance, in Figure 1 the set $\{3,6,1,7\}$ is an $A C$-component of the represented skew lattice for which $A=\{0,4\}$ and $C=\{2,5\}$. On the other hand, considering the skew lattice in Figure 4 with $A=\{1\}$ and $C=\{0\}$, the $A C$-components in this case are $\{a\},\{b\}$ and $\{c\}$.

Proposition 3.10. Let $A>B>C$ be a categorical skew chain. Then, $B$ is a disjoint union of its $A C$-components. Every $A C$-component $B^{\prime}$ of $B$ is the disjoint union of all $A$-cosets in $B$ that are contained in $B^{\prime}$ and the disjoint union of all $C$-cosets in $B$ that are contained in $B^{\prime}$, as well as the disjoint union of all the $A C$-cosets in $B^{\prime}$. For each $A C$-component $B^{\prime}$, the union $A \cup B^{\prime} \cup C$ forms a skew chain $A>B^{\prime}>C$. In particular, $A>B>C$ is categorical if and only if $A>B^{\prime}>C$ is categorical for each $A C$-component $B^{\prime}$.

The converse of this result doesn't hold as it was shown in [10] where the authors present a categorical skew chain $A>B>C$ with one unique $A C$-coset in $B$ that is not distributive (and, therefore, not strictly categorical).

\section{Strictly categorical skew lattices and orthogonality}

Given skew lattice $\mathbf{S}$ with three equivalence classes $A, B$ and $C$ such that $B$ is comparable to both $A$ and $C$, we say that $x \in A$ is covered by a coset of $C$ in $B$ if the image set of $x$ 
in $B$ is a subset of this coset of $C$. The dual definition is similar. $A$ and $C$ are orthogonal in $B$ if and only if each $x \in A$ is covered by a coset of $C$ in $B$ and, dually, each $y \in C$ is covered by a coset of $A$ in $B$.

Proposition 4.1 ([15]). Let $\mathbf{S}$ be a skew lattice with three equivalence classes $A, B$ and $C$ such that $B$ is comparable to both $A$ and $C$. If $A$ and $C$ are orthogonal in $B$, then each coset of $A$ in $B$ has nonempty intersection with each coset of $C$ in $B$. Moreover, all such coset intersections have common cardinality.

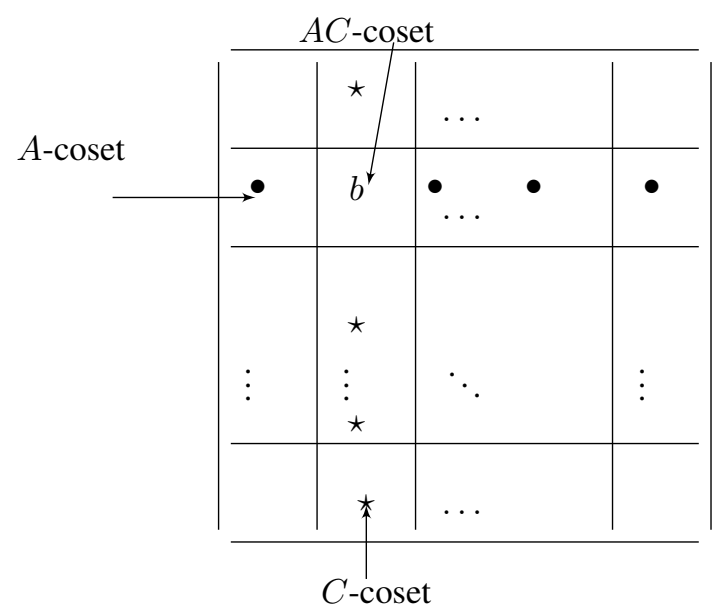

$\mathcal{D}$-class $B$

Figure 5: The partition diagram of the $\mathcal{D}$-class $B$ by $A$-cosets and $C$-cosets, when $A$ and $C$ orthogonal in $B$.

Due to Proposition 4.1, we can visualize the above $\mathcal{D}$-class $B$ in the orthogonal situation by the double partition diagram of Figure 5. We call a diagram representing intersection of partitions, like the one in Figure 5, a partition diagram. In this case, since $\mathcal{D}$-classes and cosets are rectangular skew lattices due to the rectangularity of cosets, we represent them by rectangles in this diagram. $B$ is a doubly partitioned rectangle arising from a horizontal partition by one class of congruent rectangles, the $A$-cosets, and a vertical partition by a second class of congruent rectangles, the $C$-cosets. Their nonempty intersections are again called $A C$-cosets in $B$ in conformity with [10]. It follows that if $X$ and $Y$ are respective $A$ and $C$-cosets in $B$, then

$$
|B|=\frac{|X| \cdot|Y|}{|X \cap Y|} .
$$

This makes sense in the infinite case if $|Y| /|X \cap Y|$ is understood to be the size of the class of all cosets of the form $X \cap Y$ in $Y$. Since $|X|$ and $|Y|$ are respective divisors of $|A|$ and $|C|$, it follows that if both $|A|$ and $|C|$ are finite, then $|B|$ divides $|A| \cdot|C|$ and that $|B| \leq|A| \cdot|C|$ in general. The classical case of orthogonal behavior appeared in 1993 in [15].

Proposition 4.2 ([15]). If $\mathbf{S}$ is a skew lattice with two non-comparable $\mathcal{D}$-classes $A$ and $B$, then $A$ and $B$ are orthogonal in both their meet class $M$ and in their join class $J$. The resulting $A B$-coset partitions of both $J$ and $M$ are refined by the coset partitions that $J$ 
and $M$ induce on each other. $\mathbf{S}$ is symmetric precisely when both the AB-coset partition of $M$ is the $J$-coset partition of $M$ and, dually, the $A B$-coset partition of $J$ is the $M$-coset partition of $J$. In this context, individual joins $a \vee b$ and meets $a \wedge b$ of elements $a \in A$ and $b \in B$ are determined by the orthogonality relationship.

Lemma 4.3. Let $A^{\prime}>B^{\prime}$ be a primitive subalgebra of a primitive skew lattice $A>B$ with $A^{\prime} \subseteq A$ and $B^{\prime} \subseteq B$. Then the $B^{\prime}$-cosets in $A^{\prime}$ are precisely the nonempty intersections $A^{\prime} \cap C$ of $A^{\prime}$ with some $B$-coset $C$ in $A$. Likewise, the $A^{\prime}$-cosets in $B^{\prime}$ are the nonempty intersections $B^{\prime} \cap D$ of $B^{\prime}$ with some $A$-coset $D$ in $B$.

Proof. Given $a^{\prime} \in A^{\prime}, B^{\prime} \vee a^{\prime} \vee B^{\prime} \subseteq A^{\prime} \cap\left(B \vee a^{\prime} \vee B\right)$, the intersection being nonempty. Conversely, let $A^{\prime} \cap(B \vee a \vee B)$ be nonempty with $a \in A$. Then $B \vee a \vee B=B \vee a^{\prime} \vee B$ for any $a^{\prime}$ in this intersection and hence in $A^{\prime}$. Suppose $y=b \vee a^{\prime} \vee b$ in $A^{\prime}$ for some $b \in B$. Then $y>b^{\prime}$ for some $b^{\prime}$ in $B^{\prime}$ and $b \vee a^{\prime} \vee b$ is in the same $B$-coset as $b^{\prime} \vee a^{\prime} \vee b^{\prime}$. Since both are strictly bigger than $b^{\prime}$, they must be equal. That is, $A^{\prime} \cap\left(B \vee a^{\prime} \vee B\right) \subseteq B^{\prime} \vee a^{\prime} \vee B^{\prime}$ and the first assertion follows. The second is similar.

Corollary 4.4. Let skew chain $A^{\prime}>B^{\prime}>C^{\prime}$ be a subalgebra of a skew chain $A>B>$ $C$. If the $A$-cosets and the $C$-cosets in $B$ are orthogonal, then likewise the $A^{\prime}$-cosets and the $C^{\prime}$-cosets in $B^{\prime}$ are orthogonal.

Proof. Given $c^{\prime} \in C^{\prime}$, by assumption all images of $c^{\prime}$ in $B$ lie in some $A$-coset $A \wedge b \wedge A$ in $B$. Thus all images of $c^{\prime} \in B^{\prime}$ must lie in $A \wedge b \wedge A \cap B^{\prime}$ which is an $A^{\prime}$-coset in $B^{\prime}$, due to Lemma 4.3. Likewise, given $a^{\prime} \in A^{\prime}$, its images in $B^{\prime}$ must lie in some common $C^{\prime}$-coset in $B^{\prime}$.

Clearly we now have:

Theorem 4.5. If $A>B>C$ is a skew chain for which the $A$-cosets and the $C$-cosets in $B$ are orthogonal, then $A>B>C$ is a strictly categorical skew chain, which is necessarily strictly categorical. In general, a skew lattice is strictly categorical if and only if all its skew chains have this orthogonal property.

Proof. None of the $\mathbf{X}_{n}$ or the $\mathbf{Y}_{n}$ skew chains for $n>1$ have the orthogonal property (see [9] for the construction of $\mathbf{X}_{n}$ and $\mathbf{Y}_{n}$ ). Hence by the Corollary 4.4 they cannot be subalgebras of this skew chain. It follows that $A>B>C$ is categorical.

Proposition 4.6 ([10]). Let $A>B>C$ be a strictly categorical skew chain, let $a \in A$, $c \in C$ such that $a>c$, and let $b \in B$ be the unique element such that $a>b>c$. Then, $b$ lies jointly in the $C$-coset in $B$ containing all images of $a$ in $B$ and in the $A$-coset in $B$ containing all images of $c$ in $B$.

Theorem 4.7 ([9]). Let $A>B>C$ be a strictly categorical skew chain.

(i) For any $a \in A$, there exists $b \in B$ such that $a \wedge B \wedge a \subseteq C \vee b \vee C$;

(ii) Likewise, for any $c \in C$, there exists $b^{\prime} \in B$ such that $c \vee B \vee c \subseteq A \wedge b^{\prime} \wedge A$;

(iii) Given $a>c$, then the unique $b$ in $B$ such that $a>b>c$ lies jointly in the $C$-coset in $B$ containing all images of $a$ in $B$, and in the $A$-coset in $B$ containing all images of $c$ in $B$. 
This is illustrated in Figure 5 where $b \in B$ lies in the intersection of the $A$-coset in $B$ containing all images $\bullet$ of $c \in B$ and the $C$-coset in $B$ containing all images $\star$ of $a \in B$.

Corollary 4.8 ([9]). Given any categorical skew chain $A>B>C$ of $\mathcal{D}$-classes in $\mathbf{S}$, the following statements are equivalent:

(i) $A>B>C$ is strictly categorical;

(ii) Given $a \in A, c \in C$ and a coset bijection $\chi: C \vee a \vee C \rightarrow A \wedge c \wedge A$, unique coset bijections $\varphi: B \vee a \vee B \rightarrow A \wedge b \wedge A$ and $\psi: C \vee b \vee C \rightarrow B \wedge c \wedge B$ exist such that $\chi=\psi \circ \varphi$.

We now turn our attention to the relationship between orthogonality and strict categoricity, and the consequences of this relationship in the study of the coset structure of skew chains.

Theorem 4.9. Given a skew chain $A>B>C$ in a skew lattice $\mathbf{S}, A$ and $C$ are orthogonal in $B$ if and only if $A>B>C$ is strictly categorical.

Proof. Due to Proposition 4.1, the orthogonality of $A$ and $C$ in $B$ implies that each coset of $A$ in $B$ has nonempty intersection with each coset of $C$ in $B$. Proposition 3.5 then implies that the skew chain $A>B>C$ is strictly categorical.

Conversely, let $A>B>C$ be a strictly categorical skew chain and $X$ be a order component of $A$ in $B$. Then, it follows that the orthogonality of $A$ and $C$ in $B$ is equivalent to the conditions (i) and (ii) of Theorem 4.7. Clearly, if $A$ and $C$ are orthogonal in $B$ then both (i) and (ii) hold. Conversely, assume that (i) and (ii) hold and let $K$ be a order component of $A$ in $B$. Let $x \in A$. By the assumptions, $x \wedge B \wedge x$ lies in a unique $C$-coset in $B$, say $C \vee b \vee C$ for some $b \in B$. Let $y \in K$. Then, there exists a sequence of image sets $x_{i} \wedge B \wedge x_{i}$ with $x=x_{1}, x_{2}, \ldots, x_{n}$ such that $y \in x_{n} \wedge B \wedge x_{n}$,

$$
\left(x_{i} \wedge B \wedge x_{i}\right) \cap\left(x_{j} \wedge B \wedge x_{j}\right) \neq \emptyset
$$

for every $1 \leq i, j \leq n$ and $K=\bigcup_{1 \leq i \leq n}\left(x_{i} \wedge B \wedge x_{i}\right)$. Thus

$$
\bigcap_{i \in I}\left(\left(x_{i} \wedge B \wedge x_{i}\right) \cap(C \vee b \vee C)\right) \neq \emptyset
$$

so that $K=\bigcup_{1 \leq i \leq n}\left(x_{i} \wedge B \wedge x_{i}\right) \subseteq(C \vee b \vee C)$, by Theorem 2.6. Hence, $A$ and $C$ are orthogonal in $B$.

Corollary 4.10. Let $\mathbf{S}$ be a skew Boolean algebra. Then, $A$ and $C$ are orthogonal in $B$ for all skew chains $A>B>C$ in $\mathbf{S}$.

Proof. All skew Boolean algebras are normal skew lattices by definition. Thus, they are strictly categorical skew lattices due to normality. The conclusion now follows from Theorem 4.9.

Remark 4.11. In [10], the orthogonal property for skew chains $A>B>C$ (A-cosets in $B$ and $C$-cosets in $B$ are orthogonal) is something that is added onto the categorical property. Thus, a skew lattice is strictly categorical iff it is categorical and its skew chains have the orthogonal property as in Theorem 4.5. But the orthogonal property for skew chains by itself implies that the skew lattice is categorical as in Theorem 4.9. 
We conclude this section with a weakened version of orthogonal behavior. Given a categorical skew chain $A>B>C$, the $A$-cosets in $B$ are amenable to the $C$-cosets in $B$ if in any $A C$-component $B^{\prime}$ of $B$ each $A$-coset meets each $C$-coset. Amenability is of coset nature as it is shown in the next result.

Proposition 4.12. Let $A>B>C$ be a categorical skew chain. The $A$-cosets in $B$ are amenable to the $C$-cosets in $B$ if and only if, in any $A C$-component $B^{\prime}$ of $B$, the following equivalence holds for all $x, y \in B^{\prime}$ :

$$
A \wedge x \wedge A=A \wedge u \wedge A \text { and } C \vee y \vee C=C \vee u \vee C \text { for some } u \in B^{\prime}
$$

iff

$$
A \wedge y \wedge A=A \wedge v \wedge A \text { and } C \vee x \vee C=C \vee v \vee C \text { for some } v \in B^{\prime} .
$$

Proof. Let $B^{\prime}$ be a $A C$ component of $B$ and $x, y \in B^{\prime}$. Then, $x$ and $y$ are $A C$-connected. Assume that $A \wedge x \wedge A=A \wedge u \wedge A$ and $C \vee y \vee C=C \vee u \vee C$ for some $u \in B^{\prime}$. Thus, the amenability of the $A$-cosets in $B$ to the $C$-cosets in $B$ implies the existence of $v \in(A \wedge y \wedge A) \cap(C \vee x \vee C)$ and, therefore,

$$
A \wedge y \wedge A=A \wedge v \wedge A \text { and } C \vee x \vee C=C \vee v \vee C .
$$

Conversely, let $x, y \in B$ such that $x, y$ are $A C$-connected. Suppose, without loss of generality that $u \in B$ exists such that $A \wedge x \wedge A=A \wedge u \wedge A$ and $C \vee y \vee C=C \vee u \vee C$. Thus, $A \wedge y \wedge A=A \wedge v \wedge A$ and $C \vee x \vee C=C \vee v \vee C$ for some $v \in B$. As $x$ and $y$ are arbitrary elements in $B$, each $A$-coset meets each $C$-coset in the $A C$-component of $B$ where $x$ and $y$ belong.

This is illustrated by the coset diagram in Figure 5 where the rows denote parts of $A$-cosets and the columns parts of $C$-cosets.

Proposition 4.13 ([10]). Given a categorical skew chain $A>B>C$, the A-cosets and $C$-cosets in $B$ are amenable if only only if for each $A C$-component $B^{\prime}$ of $B$, the skew chain $A>B^{\prime}>C$ is strictly categorical. Furthermore, for all $a \in A$ and $c \in C$ such that $a>c$, the unique element $b \in B^{\prime}$ such that $a>b>c$ lies jointly in the $C$-coset in $B^{\prime}$ containing all images of $a$ in $B^{\prime}$ and in the $A$-coset in $B^{\prime}$ containing all images of $c$ in $B^{\prime}$.

According to the Proposition 4.13 above, whenever $A$-cosets and $C$-cosets in $B$ are amenable in a categorical skew chain $A>B>C, A$ and $C$ are "orthogonal" in each $A C$-component $B^{\prime}$ of $B$, in the sense of Remark 4.11. Amenability, unlike orthogonality, does not by itself insure that a skew chain is categorical (cf. [10]).

Proposition 4.14 ([18]). A skew lattice is categorical if and only if all its skew chains are categorical. Furthermore, given a skew chain $A>B>C$ in a skew lattice $\mathbf{S}$, the following statements are equivalent:

(i) $\{A>B>C\}$ is categorical,

(ii) For all distinct $c, c^{\prime} \in C$,

$$
A \wedge c \wedge A=A \wedge c^{\prime} \wedge A \text { if and only if } B \wedge c \wedge B=B \wedge c^{\prime} \wedge B
$$

and, for any $b, b^{\prime}$ in some $C$-coset in $B$ such that $b>c$ and $b^{\prime}>c^{\prime}$,

$$
A \wedge b \wedge A=A \wedge b^{\prime} \wedge A
$$

[where $b^{\prime}=c^{\prime} \vee b \vee c^{\prime}$ ]. 
(iii) For all distinct $a, a^{\prime} \in A$,

$$
C \vee a \vee C=C \vee a^{\prime} \vee C \quad \text { if and only if } \quad B \vee a \vee B=B \vee a^{\prime} \vee B
$$

and, for any $b, b^{\prime}$ in some $A$-coset in $B$ such that $a>b$ and $a^{\prime}>b^{\prime}$,

$$
C \vee b \vee C=C \vee b^{\prime} \vee C
$$

[where $\left.b^{\prime}=a^{\prime} \wedge b \wedge a^{\prime}\right]$.

\section{Coset laws for distributive skew lattices}

The following results deriving from Proposition 3.7 make use of identities involving cosets to characterize categorical and strictly categorical skew lattices by the description of their coset structure. These will lead us to the recent achievements by Leech and Kinyon in [10] that can be revisited in this coset structure context permitting us a similar characterization for distributive skew lattices.

Remark 5.1. Given $A>B>C$, let $a>c$ with $a \in A$ and $c \in C$ and let $b \in B$. We can use $b$ to produce some $y \in B$ such that $a>y>c$ in two ways: either set $y=a \wedge(c \vee b \vee c) \wedge a$ or else set $y=c \vee(a \wedge b \wedge a) \vee c$. In general they need not give the same outcome.

Lemma 5.2 ([10]). A skew chain $A>B>C$ is distributive if and only if for all $a \in A$, $b \in B$ and $c \in C$ such that $a>c$,

$$
a \wedge(c \vee b \vee c) \wedge a=c \vee(a \wedge b \wedge a) \vee c
$$

When this condition holds, the common outcome is the same for all $b$ in a common $A C$ component of $B$ and is the unique element $y$ in that component such that $a>y>c$.

Theorem 5.3. Given an skew chain $A>B>C$ the following are equivalent:

(i) $A>B>C$ is distributive;

(ii) For all $A C$-components $B^{\prime}$ in $B, A>B^{\prime}>C$ is strictly categorical.

(iii) $A>B>C$ is categorical and the $A$-cosets in $B$ are amenable to the $C$-cosets in $B$.

In particular we have the following immediate consequence of Theorem 3.4:

Corollary 5.4. All strictly categorical skew chains are distributive.

Though, the converse statement to Corollary 5.4 does not hold: the skew lattice represented in Figure 2 is an example of a distributive skew lattice that is not strictly categorical having singular $A C$-components.

In the remainder of this section we turn our attention to the image set of an element in a coset and its role of a transversal of cosets permitting us to count those cosets. Transversals allows us to define the index of $B$ in $A$, first presented in [4] and denoted by $[A: B]$, as the cardinality of the image set $b \vee A \vee b$, for any $b \in B$. Dually, we define the index of $A$ in $B$, denoted by $[B: A]$, as the cardinality of the image set $a \wedge B \wedge a$, for any $a \in A$. The index $[A: B]$ equals the cardinality of the set of all $B$-cosets in $A$, and $[B: A]$ equals the cardinality of the set of all $A$-cosets in $B$. As all $A$-cosets in $B$ and all $B$-cosets in $A$ have a common size due to coset decomposition, we name this number the order of the $A$-coset in $B$ (or the order of the $B$-coset in $A$ ), denoting it by $\omega[A, B]$ or, equivalently, by $\omega[B, A]$ (denoted by $c[A, B]$ in [12]). 
Example 5.5. The situation in $A>B$ is illustrated in the following figure where the upper eggboxes represent the $B$-cosets in $A$ and the lower eggboxes represent the $A$-cosets in $B$. In this case, $|A|=18$ and $|B|=12$ with $[A, B]=3,[B, A]=2$ and $\omega[A, B]=6$.

Lemma 5.6 ([6]). Given a skew lattice $\mathbf{S}$ with comparable $\mathcal{D}$-classes $X>Y$ consider the set of all $Y$-cosets in $X,\left\{X_{i} \mid i \leq[X: Y]\right\}$ and the set of $X$-cosets in $Y,\left\{Y_{j} \mid j \leq\right.$ $[Y: X]\}$. Then, $X$ is finite if and only if $[X: Y]$ and $\omega[X, Y]$ are finite and, in that case,

$$
|X|=[X: Y] \cdot \omega[X, Y] .
$$

Similar remarks hold regarding the finitude of $Y$ and, likewise, $|Y|=[Y: X] \cdot \omega[X, Y]$ whenever $Y$ is finite.

The nature of the coset structure of a skew lattice permits such instances of combinatorial implications that are arise frequently in the literature. These combinatorial properties enabled us to derive coset laws to characterize varieties of symmetric skew lattices and cancellative skew lattices, in [15] and [4] respectively, or in the first author's research in [5, 6, 18], and his PhD thesis in [19]. Similar characterizations are also available for normal (and conormal) skew lattices, as a direct consequence of Proposition 2.9.

Proposition 5.7 ([18]). Let $\mathbf{S}$ be a skew lattice. Then $\mathbf{S}$ is normal if and only if $[B: A]=1$ and thus $\omega[A, B]=|B|$ for all comparable $\mathcal{D}$-classes $A>B$ in $\mathbf{S}$. Dually, $\mathbf{S}$ is conormal if and only if $[A: B]=1$ and thus $\omega[A, B]=|A|$ for all comparable $\mathcal{D}$-classes $A>B$ in $\mathbf{S}$.

We turn to examine the more general case of strictly categorical skew lattices. From Theorem 5.3 we are able to achieve counting results regarding distributive skew lattices.

Proposition 5.8 ([18]). Given a skew chain $A>B>C$ in a skew lattice $\mathbf{S}$ with both $A$ and $C$ finite, then

$$
|B| \geq \frac{\omega[A, B] \cdot \omega[B, C]}{\omega[A, C]} .
$$

Furthermore, $A>B>C$ is strictly categorical if and only if

$$
|B|=\frac{\omega[A, B] \cdot \omega[B, C]}{\omega[A, C]}
$$

so that in particular $B$ is also finite. With $B$ finite, $A>B>C$ is strictly categorical if and only if

$$
|B|=\omega[A, C] \cdot[B: A] \cdot[B: C] .
$$

Proposition 5.9. Let $A>B>C$ be a skew chain where $A$ and $C$ are finite. Then, $A>B>C$ is distributive if and only if

$$
\left|B_{i}\right|=\frac{\omega[A, B] \cdot \omega[B, C]}{\omega[A, C]}
$$

for each $A C$-component $B_{i}$ of $B$.

Corollary 5.10. Let $A>B>C$ be a skew chain where $A$ and $C$ are finite. If $B$ has $n<\infty A C$-components, then $A>B>C$ is distributive if and only if

$$
|B|=n \frac{\omega[A, B] \cdot \omega[B, C]}{\omega[A, C]} .
$$




\section{References}

[1] R. J. Bignall and J. E. Leech, Skew Boolean algebras and discriminator varieties, Algebra Universalis 33 (1995), 387-398, doi:10.1007/bf01190707.

[2] K. Cvetko-Vah, Skew Lattices in Rings, Ph.D. thesis, University of Ljubljana, Slovenia, 2005.

[3] K. Cvetko-Vah, A new proof of Spinks' theorem, Semigroup Forum 73 (2006), 267-272, doi: 10.1007/s00233-006-0603-8.

[4] K. Cvetko-Vah, M. Kinyon, J. Leech and M. Spinks, Cancellation in skew lattices, Order 28 (2011), 9-32, doi:10.1007/s11083-010-9151-7.

[5] K. Cvetko-Vah and J. Pita Costa, On the coset laws for skew lattices in rings, Novi Sad J. Math. 40 (2010), 11-25.

[6] K. Cvetko-Vah and J. Pita Costa, On the coset laws for skew lattices, Semigroup Forum 83 (2011), 395-411, doi:10.1007/s00233-011-9325-7.

[7] J. A. Green, On the structure of semigroups, Ann. Math. 54 (1951), 163-172, doi:10.2307/ 1969317.

[8] J. M. Howie, An Introduction to Semigroup Theory, volume 7 of London Mathematical Society Monographs, Academic Press, London, 1976.

[9] M. Kinyon and J. Leech, Categorical skew lattices, Order 30 (2013), 763-777, doi:10.1007/ s11083-012-9275-z.

[10] M. Kinyon, J. Leech and J. Pita Costa, Distributivity in skew lattices, Semigroup Forum 91 (2015), 378-400, doi:10.1007/s00233-015-9722-4.

[11] J. Leech, Towards a theory of noncommutative lattices, Semigroup Forum 34 (1986), 117-120, doi:10.1007/bf02573155.

[12] J. Leech, Skew lattices in rings, Algebra Universalis 26 (1989), 48-72, doi:10.1007/ bf01243872.

[13] J. Leech, Skew Boolean algebras, Algebra Universalis 27 (1990), 497-506, doi:10.1007/ bf01188995.

[14] J. Leech, Normal skew lattices, Semigroup Forum 44 (1992), 1-8, doi:10.1007/bf02574320.

[15] J. Leech, The geometric structure of skew lattices, Trans. Amer. Math. Soc. 335 (1993), $823-$ 842, doi:10.2307/2154407.

[16] J. Leech, Recent developments in the theory of skew lattices, Semigroup Forum 52 (1996), 7-24, doi:10.1007/bf02574077.

[17] J. Pita Costa, On the coset structure of a skew lattice, Demonstratio Math. 44 (2011), 673-692, doi:10.1515/dema-2013-0338.

[18] J. Pita Costa, Coset laws for categorical skew lattices, Algebra Universalis 68 (2012), 75-89, doi:10.1007/s00012-012-0194-z.

[19] J. Pita Costa, On the Coset Structure of Skew Lattices, Ph.D. thesis, University of Ljubljana, Slovenia, 2012.

[20] M. Spinks, On middle distributivity for skew lattices, Semigroup Forum 61 (2000), 341-345, doi:10.1007/p100006032. 